University of Texas Rio Grande Valley

ScholarWorks @ UTRGV

Psychological Science Faculty Publications and

Presentations

College of Liberal Arts

$11-2020$

\title{
Perceived Burdensomeness and Thwarted Belongingness Influence the Childhood Polyvictimization and Suicide Ideation Association among Hispanic Undergraduates
}

\author{
Claire Hsieh \\ The University of Texas Rio Grande Valley \\ Ruby Charak \\ The University of Texas Rio Grande Valley, ruby.charak@utrgv.edu \\ Ines Cano-Gonzalez \\ The University of Texas Rio Grande Valley \\ Leo Gonzalez \\ Michelle E. Roley \\ The Ohio State University
}

See next page for additional authors

Follow this and additional works at: https://scholarworks.utrgv.edu/psy_fac

Part of the Psychology Commons

\begin{abstract}
Recommended Citation
Claire Hsieh, Ruby Charak, Ines Cano-Gonzalez, Leo Gonzalez, Michelle E. Roley-Roberts \& Joseph D. Hovey (2020) Perceived burdensomeness and thwarted belongingness influence the childhood polyvictimization and suicide ideation association among Hispanic undergraduates, Death Studies, DOI: 10.1080/07481187.2020.1846227
\end{abstract}

This Article is brought to you for free and open access by the College of Liberal Arts at ScholarWorks @ UTRGV. It has been accepted for inclusion in Psychological Science Faculty Publications and Presentations by an authorized administrator of ScholarWorks @ UTRGV. For more information, please contact justin.white@utrgv.edu, william.flores01@utrgv.edu. 


\section{Authors}

Claire Hsieh, Ruby Charak, Ines Cano-Gonzalez, Leo Gonzalez, Michelle E. Roley, and Joseph D. Hovey 
CHILDHOOD POLYVICTIMIZATION AND SUICIDE IDEATION

\title{
Perceived Burdensomeness and Thwarted Belongingness Influence the Childhood
}

\section{Polyvictimization and Suicide Ideation Association among Hispanic Undergraduates}

\author{
Claire Hsieh, B.S. \\ Ruby Charak, Ph.D. \\ Ines Cano-Gonzalez, M.A. \\ Department of Psychological Science
}

The University of Texas Rio Grande Valley, Edinburg, Texas.

Leo Gonzalez, M.A.

Department of Family and Community Medicine

The University of Texas Southwestern Medical Center

Michelle E. Roley-Roberts, Ph.D.

Department of Psychiatry

CHI Health Creighton University, Omaha, Nebraska

Joseph D. Hovey, Ph.D.

Department of Psychological Science

The University of Texas Rio Grande Valley, Edinburg, Texas.

Author for correspondence: Ruby Charak, Ph.D., Associate Professor, Department of

Psychological Science, EIEAB 3.207, The University of Texas Rio Grande Valley, 1201 W.

University Dr., Edinburg, Texas 78539. Ph: +1-956-665-3733. Email: charakruby@gmail.com or ruby.charak@utrgv.edu

APA citation: Hsieh, C., Charak R., Cano-Gonzalez, I., Gonzalez, L., Roley-Roberts, M. E., \& Hovey, J. D. (2020). Perceived burdensomeness and thwarted belongingness influence the childhood polyvictimization and suicide ideation association among Hispanic undergraduates. Death Studies. Advance online publication. doi.org/10.1080/07481187.2020.1846227 


\title{
CHILDHOOD POLYVICTIMIZATION AND SUICIDE IDEATION
}

\begin{abstract}
The present study examined the mediating effect of perceived burdensomeness (PB) and thwarted belongingness (TB) in the association between childhood polyvictimization and suicide ideation (past week) among 528 Hispanic college students. Nearly 10\% reported polyvictimization, $19.8 \%$ had suicide ideation, and polyvictimization was a risk factor of suicide ideation through PB and TB. The indirect effect through PB was stronger than the indirect effect through TB. Interventions should focus on PB and TB to alleviate suicide ideation among Hispanic undergraduate students.
\end{abstract}

Keywords. Polyvictimization, suicide, Interpersonal psychological theory of suicide, Hispanic 
CHILDHOOD POLYVICTIMIZATION AND SUICIDE IDEATION

\section{Perceived Burdensomeness and Thwarted Belongingness Influence the Childhood}

\section{Polyvictimization and Suicide Ideation Association among Hispanic Undergraduates}

Hispanics in the U.S. face structural barriers, such as deficits in mental and social services, poverty, inequities and discriminatory practices that negatively impact their mental health and wellbeing (Leong \& Leach, 2008; Molina et al., 2016). In 2017, suicide was the third leading cause of death in Hispanics aged 15-34 years (Li et al., 2019). Additionally, the nonHispanic White population is twice as likely to receive mental health services than the Hispanic population (Substance Abuse and Mental Health Services Administration [SAMSHA], 2019).

Suicide ideation, that is, thoughts of killing oneself, is a strong risk factor for suicide attempts and there is an urgent call for interventions targeting suicide ideation (Jobes \& Joiner, 2019). Some U.S. Hispanic populations have historically had fewer risks of negative health outcomes and mortality, including suicidal behavior and death by suicide, despite socioeconomic disadvantages, a phenomenon known as the "Latino paradox" (Silva \& Van Orden, 2017). However, suicide risk within Hispanic communities have been sharply increasing since 2000 (Silva \& Van Orden, 2017). Although the Collaborative Psychiatric Epidemiological Surveys estimated that the lifetime prevalence of suicide ideation (11.35\%) among Hispanics was lower as compared to non-Hispanic Whites (16.10\%) (Borges et al., 2012; Silva \& Van Orden, 2017), rates of suicide ideation and attempt may be severely underreported as a result of limited access to health coverage and mental health screening as well as cultural stigmas toward suicide and mental health in Hispanic culture (Brenes, 2019; Dobalian \& Rivers, 2007). Examining risk factors of suicide ideation in Hispanic populations is important for providing early, culturally inclusive interventions to alleviate rates of suicide ideation and avert suicide attempts. 


\section{CHILDHOOD POLYVICTIMIZATION AND SUICIDE IDEATION}

A known risk factor of suicidal behavior is exposure to childhood adversities, including polyvictimization (Charak et al., 2016; Ford et al., 2018; Finkelhor et al., 2009).

Polyvictimization refers to multiple experiences of different types of victimization, such as peer/sibling victimization, witnessing family violence, conventional crime, sexual victimization, and child maltreatment (Finklehor et al., 2009; Finklehor et al., 2011). Lifetime polyvictimization in adults is defined as having exposure to 15 or more types of childhood traumatic events from a total of 34 different types of childhood adversities (Finkelhor et al., 2009). Although polyvictimization can range from cutoff scores of 9 to 12 for children aged 3 to 14, the cutoff score of 15 or more has been established by Finkelhor et al. (2009) and supported through victimization literature for youth aged 15 to 18 , as well the most appropriate cutoff score to determine lifetime polyvictimization in adults.

In a U.S. national survey, $10.6 \%$ of children and adolescents had exposure to 9 or more victimization types across their lifespan (Finkelhor et al., 2009). In the same national survey, $12.3 \%$ of the Hispanic youth experienced 10 or more adverse events (Turner et al., 2010), indicating Hispanic youths' increased risk of experiencing polyvictimization. Prior studies demonstrate that adverse childhood experiences of any type attribute to suicide ideation and other negative mental health outcomes, and results in a 2-to-5-fold increase in the risk of attempted suicide during childhood/adolescence and adulthood (Afifi et al., 2008). Most studies on childhood polyvictimization focus on short-term outcomes in youth or adolescents (e.g., Charak et al., 2019; Karsberg et al., 2019). The limited research on the impact of polyvictimization suggest greater risk for suicidal behavior in adulthood (e.g., Afifi et al., 2016; Charak et al., 2016). 


\section{CHILDHOOD POLYVICTIMIZATION AND SUICIDE IDEATION}

Mechanisms operating between childhood polyvictimization and suicide ideation have been theorized within the interpersonal psychological theory of suicide (IPTS; Van Orden et al., 2010). IPTS suggests that suicide ideation results from feeling socially alienated and disconnected from others, a phenomenon known as thwarted belongingness (TB), and when an individual has a constant misperception of being a burden on others, a phenomenon known as perceived burdensomeness (PB) (Joiner, 2007). Literature addressing suicide ideation contain partial support for stronger associations of suicide ideation with PB compared to the association between suicide ideation and TB (Bryan et al., 2010; Chu et al., 2016; Van Orden et al., 2008). A meta-analysis involving 122 samples indicated a positive association between $\mathrm{PB}(r=.48)$, TB $(r$ $=.37)$, and suicide risk ( $\mathrm{PB} r=.42$; $\mathrm{TB} r=.33$ ) suggesting that higher $\mathrm{PB}$ and $\mathrm{TB}$ were linked with greater risk of suicide (Chu et al., 2017). Findings from an inpatient sample of adults from Mexico found that the interaction between PB and TB significantly predicted suicidal behaviors (Hurtado et al., 2019) with PB being the most critical predictor of suicidal ideation. Due to limited research providing support for the IPTS within U.S. Hispanic adults, more studies are needed to examine IPTS to inform clinical interventions.

Studies on childhood maltreatment and IPTS suggest that childhood emotional maltreatment is associated with the development of $\mathrm{PB}$ and $\mathrm{TB}$, which contribute to suicidal behavior (Joiner 2007; Puzia et al., 2014; Schönfelder et al., 2019; Van Orden et al. 2010). Youth who have experienced victimization may feel different and seclude themselves from others in an attempt to maintain safety, or feel disconnected with their peers (Miller et al., 2013). Experiencing childhood victimization often creates cognitive and emotional distortions around self-concept and world views, leading to feelings of low self-worth and interpersonal difficulties, which may in turn, contribute to TB and PB (Miller et al., 2013). Studies also suggest that 


\section{CHILDHOOD POLYVICTIMIZATION AND SUICIDE IDEATION}

emotional maltreatment and other forms of childhood adversities increase the likelihood of suicidal ideation and its transition into the capacity of attempting suicide (Miller et al., 2016; Nguyen et al., 2019; Roley-Roberts et al., 2017; Van Orden et al., 2010). A meta-analysis of 68 studies indicated that exposure to childhood physical, sexual or emotional abuse was associated with 2-to-3-fold increased risk of suicidal ideation and attempts during adulthood (Angelakis et al., 2019). Studies examining risk factors of suicide ideation have started to recognize the additive model of childhood victimization and could be informative in examining the effect of childhood polyvictimization.

Based on the IPTS framework, the present study examined the role of PB and TB in the association between childhood polyvictimization and suicide ideation in a sample of Hispanic undergraduate students. First, we hypothesized that childhood polyvictimization would have a significant indirect effect on suicide ideation via PB and TB. Second, we hypothesized that the strength of the indirect effect of childhood polyvictimization on suicide ideation would be stronger via PB than through TB (Bryan, 2010).

\section{Participants}

\section{Method}

Participants were 528 Hispanic emerging adults consisting of 369 women (69.9\%), 159 men $(30.1 \%)$ between ages 18 to $29(M=20.13, S D=2.4)$, attending a University in South Texas. The inclusionary criteria in the present study were (i) age range of 18-29 years, (ii) a U.S. citizen or legal resident, and (iii) self-identifying as Hispanic. Self-reported country of origin of the 528 consisted of 412 from Mexico (77.9\%), 39 from the U.S. (7.4\%), 17 (3.2\%) from other countries (i.e., Argentina, Honduras, and Spain), and 73 participants did not specify. Nearly $30 \%$ indicated being in a relationship ( $n=161$; either married, cohabitating or in a relationship) and $70 \%$ indicated not having a partner ( $n=367$; either single, separated, divorce or widowed). 


\section{CHILDHOOD POLYVICTIMIZATION AND SUICIDE IDEATION}

Nearly $60 \%(n=312)$ reported annual household income of less than $\$ 40,000$, with $13 \%(n=69)$ reporting a yearly income of less than $\$ 10,000$. Most participants $(87.9 \%, n=464)$ identified as heterosexual while $12.1 \%(n=64)$ identified as a sexual minority.

\section{Measures}

The Juvenile Victimization Questionnaire-Second Revision (JVQ-R2; Finkelhor et al., 2011) gathers a range of childhood and adolescent victimization types that occurred before age 18. Comprising 34 offenses within the U.S. Federal Government in the National Crime Victimization Survey (Rennison \& Rand, 2003), JVQ-R2 gathers information regarding five general domains: conventional crime, child maltreatment, peer and sibling victimization, sexual victimization, and witnessing and indirect victimization. They are scored in a dichotomous variable, 0 for "no" or absence of the experience and 1 for "yes" or endorsement of the experience. In the present study, a dichotomous score of 0 for "no childhood polyvictimization" (i.e., score of 0-14) and 1 for "polyvictimization present" (i.e., score of 15-34) was created to measure lifetime polyvictimization (Finkelhor et al., 2009). Furthermore, experiencing 15 to 19 adverse experiences signified low polyvictimization, experiencing 20 to 34 adverse events signified high polyvictimization, as categorized in prior literature (Sterzing et al., 2019). JVQ-R2 is a suitable measure for identifying experiences of victimization in clinical samples (ÁlvarezLister et al., 2014), community samples, and with Hispanic samples (Sabina et al., 2016). Cronbach's alpha for the present study is .874 .

The Interpersonal Needs Questionnaire (INQ-15; Van Orden et al., 2012) assesses PB with six items and TB with nine items, rated on a 7-point scale (not at all true for me $=1$ to very true for me $=7$ ). TB assesses perceived social isolation through reverse-coded statements of positive experiences, such as "these days, I feel like I belong." Suggested cut-off scores of 17 for 


\section{CHILDHOOD POLYVICTIMIZATION AND SUICIDE IDEATION}

PB and 37 for TB classify high-risk participants (Brookings \& Pederson, 2019). The reliability of INQ has been excellent in a sample of Hispanic adults (PB: $\alpha=.95$, and TB: $\alpha=.88$ ) (Silva et al., 2018). In the present study, $\alpha=.959$ for PB and .733 for TB.

Beck Scale for Suicide Ideation (BSS; [Beck et al., 1988]) is 21 items measuring selfreported suicide ideation of which the first 19 items are scored; the last two items record past suicide attempts. BSS inquires for suicide ideation and attempts during the past one week. Each of the 19 items are on a 3-point such that $0=$ None (i.e., I rarely or only occasionally think about killing myself), 1 = Weak (i.e., I have frequent thoughts about killing myself) and $2=$ Moderate to strong (i.e., I continuously think about killing myself). Total scores ranging from 0 to 38 are obtained through the summation of the item values. The first 5 items (i.e., "wish to live," "wish to die," "reasons for living or dying," "active suicide attempt," and "passive suicide attempt) screen for suicidal ideation. Participants reporting 0 on suicide ideation items skip to the end of the scale, receive a zero on the 19-items used in the present study, and complete the last two items about past suicide attempts (not included in the present study). Although there is no clinically established cut-off score, BSS authors suggest categorizing any positive score as “elevated risk" (Beck \& Steer, 1991), others apply a cut-off of 24 or greater as "imminent risk" (Cukrowicz et al., 2011). BSS was found to have a good internal consistency $(\alpha=.95)$ in a sample with a 57\% Latinos (Cheref et al., 2014). Cronbach's alpha in the present study was .873.

\section{Procedure}

Students enrolled in psychology courses at a university in south Texas were recruited to participate in an online survey via SONA, an automated undergraduate student participatory pool. On average, students took 45 minutes to complete the survey. Participants who completed the online survey received course credit if enrolled in the General Psychology course, and 


\section{CHILDHOOD POLYVICTIMIZATION AND SUICIDE IDEATION}

students enrolled in other psychology courses received extra-credit at the discretion of their instructor. Data collection was conducted between Spring 2017 and Summer 2019. At the end of the survey, students received a debriefing form that contained contact information of regional and national social services providing crisis intervention and clinical services. The Institutional Review Board at the corresponding author's institution approved the research study.

\section{Statistical Analyses}

After conducting bivariate correlation, mediation analysis using Mplus version 8.0 evaluated the indirect effects of childhood polyvictimization on suicidal ideation via PB and TB. In this mediation analysis, we added gender and age as covariates controlling for their effects on suicide ideation; and relationship status as a covariate controlling for its effect on TB. The magnitude of indirect effects was examined using the product-of-coefficient approach to calculate standard errors. Bias-corrected bootstrapping procedures for confidence intervals with a total of 5,000 bootstrapped samples were used to corroborate findings from the product-ofcoefficient tests (Preacher \& Hayes, 2008). In the present study, a 95\% confidence interval not containing a zero was considered statistically significant. Third, all significant indirect effects of childhood polyvictimization on suicide ideation via PB and TB were compared, with the Model constraint option in Mplus calculating a Wald test to examine differences between parameters under consideration using bootstrapping ( $N=10,000$ iterations). The present study did not have missing values since we used the forced response option in Qualtrics during data collection. However, the participants were free to leave the study at any point without any penalty.

\section{Results}

Some of these students $(n=46,8.7 \%)$ experienced polyvictimization, that is, they had a score of 15 or above on the JVQ-R2. Of these, 31 (5.9\%) experienced low polyvictimization and 


\section{CHILDHOOD POLYVICTIMIZATION AND SUICIDE IDEATION}

15 (2.8\%) had high polyvictimization. Findings indicated a high risk of PB above the cut-off of $17(n=88 ; 14.4 \%)$ and TB above the cut-off of $37(n=111 ; 19.3 \%)$ among the participants.

Nearly $20 \%(n=118 ; 19.8 \%)$ experienced elevated levels of suicide ideation, and $10 \%(n=53)$ reported clinically significant suicidal ideation (Beck \& Steer, 1991; Cochrane-Brink et al., 2000; Cukrowicz et al., 2011).

Bivariate correlations demonstrated that all study variables were significantly and positively correlated (Table 1). The magnitude of correlation between polyvictimization, $\mathrm{PB}, \mathrm{TB}$, and suicide ideation was low to moderate. In the mediation model, there was no significant direct effect between childhood polyvictimization and suicidal ideation (path $c$; Figure 1). However, childhood polyvictimization had significant indirect effects on suicide ideation through PB and TB (Table 2). When the two indirect effects were compared, the indirect effect of childhood polyvictimization on suicide ideation through PB was stronger $(z=1.550$, S.E. $=.470, p<.001)$ than the indirect effect of childhood polyvictimization through TB.

\section{Discussion}

In the present study, $8.7 \%$ of the Hispanic undergraduate students reported exposure to childhood polyvictimization, which is slightly lower than the $10.6 \%$ polyvictimization found across all age groups (Finkelhor et al., 2009), and 12.3\% found among Hispanic children/adolescents (Turner et al., 2010). Notably, our study uses a more stringent cut off score (>14 adverse events) compared to Turner et al. (2010) who used a cut-off exposure to 10 childhood adverse events. Although various cut-off scores are used to identify polyvictimization (i.e., $9+$ in children aged 3 to $6,10+$ in children aged 7 to $10,12+$ in youth aged 11 to 14 ), 15 or more is suggested for adults, and as the current study measures emerging adults' experiences of childhood polyvictimization in their lifetime, we chose the upper bound cut-off (Finkelhor et al., 


\section{CHILDHOOD POLYVICTIMIZATION AND SUICIDE IDEATION}

2009). Nearly one in five (19.8\%) participants experienced elevated levels of suicide ideation, with $10 \%$ being at a clinically significant risk (Beck \& Steer, 1991; Cukrowicz et al., 2011; Fitzpatrick et al., 2005). Our findings are in line with a previous large sample size study on college students wherein the rate of suicide ideation was $19.1 \%$ among Hispanic undergraduate students and $24 \%$ across the total sample (Liu et al., 2019).

Although the present study did not find evidence for the significant direct effect of childhood polyvictimization on suicide ideation, there were significant indirect effects of childhood polyvictimization on suicide ideation through PB and TB. To the best of our knowledge, the present findings are the first to be found in a Hispanic sample of college undergraduate students. Previously studies have demonstrated the effect of childhood maltreatment leading to PB and TB in non-Hispanic White samples (Cero \& Sifers, 2013; LevWiesel \& Sternberg, 2012; Van Orden et al., 2010), and also found a non-significant direct effect between childhood maltreatment types and suicide ideation (Schönefelder et al., 2019). Our study findings suggest that exposure to multiple childhood adversities influence the development of $\mathrm{PB}$ and $\mathrm{TB}$, leading to greater risk of suicide ideation. Clinical interventions for reducing suicide ideation should be directed towards children and adolescents with exposure to polyvictimization, as these developmental stages could prove to be vital in prevention of suicide ideation.

Furthermore, our finding that PB has a greater magnitude than TB in the association between childhood polyvictimization and suicide ideation is in line with prior studies. Previously, PB has demonstrated greater significance than TB in affecting a multitude of mental health outcomes, including suicidal behavior (Bryan, 2010; Roeder \& Cole, 2018). When tested separately in prior studies, PB and TB were both significant predictors of suicide ideation in 


\section{CHILDHOOD POLYVICTIMIZATION AND SUICIDE IDEATION}

cross sectional analyses and longitudinally; however, when tested together only PB was significant cross sectionally (Roeder \& Cole, 2018). Another study consisting of a military sample, displayed similar findings wherein PB and TB had significant associations with suicide ideation, with PB displaying a stronger association with suicide ideation than TB. Additionally, research consistently demonstrates a predictive value of PB on suicide ideation/attempt, whereas there are mixed findings for the effects of TB on suicide ideation (Hill \& Pettit, 2014; Ma et al., 2016; Van Orden et al., 2008). Oakley-Frost and colleagues (2019) also reported a significant indirect effect of ethnic identity through $\mathrm{PB}$ but not via TB, on suicide risk in Hispanic/Latinx students, which is in line with prior literature with the effect of PB on suicide ideation being higher than TB (Hill \& Pettit, 2014) in Hispanic samples.

The present findings may be interpreted in context of the core values of the Hispanic culture. Familismo, a core cultural value in the Hispanic culture, lays strong emphases on familial solidarity, attachment, loyalty and responsibility towards family and extended family members (Consoli \& Llamas, 2013). However, being in a volatile environment with exposure to childhood polyvictimization threatens familismo (Kennedy \& Ceballo, 2013), which can enhance the risk of self-blame and feelings of worthlessness and/or being a burden on the family, translating into the risk factors of PB and TB (Calzada et al., 2012). Although the present study did not explicitly examine familismo, sociocultural contexts may influence $\mathrm{PB}$ and $\mathrm{TB}$ for Hispanic young adults living on the Mexico-South Texas border of the U.S, highlighting the need to further explore these factors. Furthermore, acculturation may be a relevant factor to consider in the present findings. Acculturation describes the superimposition of one culture onto another, subsequent expectations to assimilate to that culture, and the negative repercussions that fall upon the suppressed group (Escobar \& Vega, 2000). Prior literature observing acculturation, 


\section{CHILDHOOD POLYVICTIMIZATION AND SUICIDE IDEATION}

IPTS, and suicide behavior are similar to the current study findings, reporting an indirect effect between ethnic identity and suicide risk through PB (Oakey-Frost et al., 2019). The current study does not measure acculturation; however, acculturation may have a contributing role in the current study findings and future studies should investigate the nuances of acculturation, IPTS, and suicidal behavior among Hispanic adults.

Limitations include, first, a convenience sample of self-identifying Hispanic participants from the same university, a majority of who were of Mexican descent, thus may not represent Hispanic young adults nationally (Salinas \& Lozano, 2019). Second, the self-reported questionnaires contained sensitive information about suicidal behavior, childhood victimization experiences, and negative psychological outcomes, which may cause some distress (Brener et al., 2003). However, online data collection forums may paradoxically increase the likelihood of reporting sensitive information in the absence of an interviewer (Tourangeau \& Smith, 1996). Additionally, the measures of childhood victimization may not adequately capture the victimizations experiences of Hispanic youth who are uniquely at risk for separation from family members, and whose family members are at risk for deportation (Flores \& Salazar, 2017). Recall bias may also be present during reporting of childhood victimization experiences. Third, the present study is a cross-sectional design and temporal sequencing of variables is based on the IPTS frameworks. This model should be examined in a longitudinal design with the inclusion of exposure to other lifetime adversities (e.g., intimate partner violence, financial loss) and their association with facets of IPTS.

Aside from the underrepresentation in literature, Hispanics also face deficits in access to mental healthcare services (Lanier et al., 2014; Leong, \& Leach, 2008) and deficits in culturally modified interventions geared toward their wellbeing. Improving accessibility of services and 


\section{CHILDHOOD POLYVICTIMIZATION AND SUICIDE IDEATION}

modifying interventions to make them culturally appropriate will help in grasping the negative mental health outcomes in racial/ethnic minorities and developing specifically tailored interventions. Fostering a stronger ethnic identity among Hispanic clients has previously been found to alleviate suicide behavior (Oakey-Frost et al., 2019) and preventative programs tailored towards strengthening ethnic identity should be developed aimed at improving social connectedness and social capital to reduce rates of suicidal behavior. As perceived burdensomeness and thwarted belongingness are dynamic cognitive and affective states amendable to change (Hill \& Pettit, 2014; Van Orden et al., 2010), targeting feelings and thoughts of thwarted belongingness and burdensomeness through cognitive restructuring should be prioritized to alleviate suicide ideation. This may also increase engagement with clinical services by highlighting the clinician as a source of social support and can improve therapeutic alliance and wellbeing of the client (Calear et al., 2014). Behavioral activation approaches geared towards activities that increase feelings of connectedness should also be implemented (Joiner et al., 2009).

To summarize, the present findings are important in so much that they emphasize the role of feelings of thwarted belongingness and perceived burdensomeness as important factors contributing to the association between childhood polyvictimization and suicide ideation in adulthood among Hispanic college students. Future studies should take into consideration cultural — ethnic identification, acculturation, familismo - and contextual factors — access to mental health services, improving cultural competencies of therapists-when studying the association between victimization, suicide ideation and components of the interpersonal theory of suicide. 


\section{CHILDHOOD POLYVICTIMIZATION AND SUICIDE IDEATION}

\section{References}

Afifi, T. O., Enns, M. W., Cox, B. J., Asmundson, G. J., Stein, M. B., \& Sareen, J. (2008). Population attributable fractions of psychiatric disorders and suicide ideation and attempts associated with adverse childhood experiences. American Journal of Public Health, 98(5), 946-952.

Álvarez-Lister, M. S., Pereda, N., Abad, J., \& Guilera, G., \& GReVIA. (2014). Polyvictimization and its relationship to symptoms of psychopathology in a southern European sample of adolescent outpatients. Child Abuse and Neglect, 38(4), 747-756.

Angelakis I., Gillespie, E. L., \& Panagioti, M. (2019). Childhood maltreatment and adult suicidality: a comprehensive systematic review with meta-analysis. Psychological Medicine, 49, 1057-1078.

Beck, A. T., \& Steer, R. A. (1991). Relationship between the Beck anxiety inventory and the Hamilton anxiety rating scale with anxious outpatients. Journal of Anxiety Disorders, 5(3), 213-223.

Beck, A. T., Steer, R. A., \& Ranieri, W. F. (1988). Scale for suicide ideation: Psychometric properties of a self-report version. Journal of Clinical Psychology, 44(4), 499-505.

Borges, G., Orozco, R., Rafful, C., Miller, E., \& Breslau, J. (2012). Suicidality, ethnicity and immigration in the USA. Psychological Medicine, 42(6), 1175-1184. https://doi.org/10.1017/S0033291711002340

Brener, N. D., Billy, J. O., \& Grady, W. R. (2003). Assessment of factors affecting the validity of self-reported health-risk behavior among adolescents: evidence from the scientific literature. Journal of Adolescent Health, 33(6), 436-457. 


\section{CHILDHOOD POLYVICTIMIZATION AND SUICIDE IDEATION}

Brenes, F. (2019). Hispanics, mental health, and suicide: Brief report. Hispanic Health Care International, 17(3), 133-136. https://doi.org/10.1177/1540415319843072

Brookings, J. B., \& Pederson, C. L. (2019). INQ-15 cut-off scores for suicidal ideation screening of women with chronic, invisible illnesses. Clinical Psychology and Psychotherapy, $26(2), 273-275$

Bryan, C. J., Morrow, C. E., Anestis, M. D., \& Joiner, T. E. (2010). A preliminary test of the interpersonal-psychological theory of suicidal behavior in a military sample. Personality and Individual Differences, 48(3), 347-350.

Calear, A. L., Batterham, P. J., \& Christensen, H. (2014). Predictors of help-seeking for suicidal ideation in the community: risks and opportunities for public suicide prevention campaigns. Psychiatry Research, 219(3), 525-530.

Calzada, E. J., Tamis-LeMonda, C. S., \& Yoshikawa, H. (2012). Familismo in Mexican and Dominican families from low-income, urban communities. Journal of Family Issues, 34 (12), 1696-1724.

Cero, I., \& Sifers, S. (2013). Moderating factors in the path from physical abuse to attempted suicide in adolescents: Application of the Interpersonal-Psychological Theory of Suicide. Suicide and Life-Threatening Behavior, 43(3), 296-304.

Charak, R., Byllesby, B. M., Roley, M. E., Claycomb, M. A., Durham, T. A., Ross, J., Armour, C., \& Elhai, J. D. (2016). Latent classes of childhood poly-victimization and associations with suicidal behavior among adult trauma victims: Moderating role of anger. Child Abuse and Neglect, 62, 19-28.

Charak, R., Ford, J. D., Modrowski, C. A., \& Kerig, P. K. (2019). Polyvictimization, emotion dysregulation, symptoms of posttraumatic stress disorder, and behavioral health problems 


\section{CHILDHOOD POLYVICTIMIZATION AND SUICIDE IDEATION}

among justice-involved youth: A latent class analysis. Journal of Abnormal Child Psychology, 47(2), 287-298.

Chu, C., Buchman-Schmitt, J. M., Stanley, I. H., Hom, M. A., Tucker, R. P., Hagan, C. R., Rogers, M. L., Podlogar, M. C., Chiurliza, B., Patrocs, C. H. G., \& Joiner, T. E. (2017). The interpersonal theory of suicide: A systematic review and meta-analysis of a decade of cross-national research. Psychological Bulletin, 143(12), 1313-1345.

Chu, C., Rogers, M. L., \& Joiner, T. E. (2016). Cross-sectional and temporal association between non-suicidal self-injury and suicidal ideation in young adults: The explanatory roles of thwarted belongingness and perceived burdensomeness. Psychiatry Research, 246, 573-580.

Cochrane-Brink, K. A., Lofchy, J. S., \& Sakinofsky, I. (2000). Clinical rating scales in suicide risk assessment. General Hospital Psychiatry, 22(6), 445-451.

Consoli, M. L. M., \& Llamas, J. D. (2013). The relationship between mexican american cultural values and resilience among mexican american college students: A mixed methods study. Journal of Counseling Psychology, 60(4), 617-624.

Cukrowicz, K. C., Schlegel, E. F., Smith, P. N., Jacobs, M. P., Van Orden, K. A., Paukert, A. L., Pettit, J. W., \& Joiner, T. E. (2011). Suicide ideation among college students evidencing subclinical depression. Journal of American College Health, 59(7), 575-581.

Dobalian, A., \& Rivers, P. A. (2007). Racial and Ethnic Disparities in the Use of Mental Health Services. The Journal of Behavioral Health Services \& Research, 35(2), 128-141. doi:10.1007/s11414-007-9097-8 


\section{CHILDHOOD POLYVICTIMIZATION AND SUICIDE IDEATION}

Escobar, J. I., \& Vega, W. A. (2000). Mental health and immigration's AAAs: where are we and where do we go from here? The Journal of Nervous and Mental Disease, 188(11), 736740. https://doi.org/10.1097/00005053-200011000-00003

Finkelhor, D., Ormrod, R. K., \& Turner, H. A. (2009). Lifetime assessment of poly-victimization in a national sample of children and youth. Child Abuse and Neglect, 33(7), 403-411.

Finkelhor, D., Turner, H., Hamby, S., \& Ormrod, R. (2011). Poly-victimization: Children's exposure to multiple types of violence, crime, and abuse (pgs. 1-12). Juvenile Justice Bulletin - NCJ 235504. U.S. Government Printing Office.

Fitzpatrick, K. K., Witte, T. K., \& Schmidt, N. B. (2005). Randomized controlled trial of a brief problem-orientation intervention for suicidal ideation. Behavior Therapy, 36(4), 323-333.

Flores, G., \& Salazar, J. C. (2017). Immigrant Latino children and the limits of questionnaires in capturing adverse childhood events. Pediatrics 140(5). doi.org/10.1542/peds.2017-2842

Ford, J., Charak, R., Modriowski, C. A., \& Kerig, P. K. (2018). PTSD and dissociation symptoms as mediators of the relationship between polyvictimization and psychosocial and behavioral problems among justice-involved adolescents. Journal of Trauma and Dissociation, 19(3), 325-346.

Hill, R. M., \& Pettit, J. W. (2014). Perceived burdensomeness and suicide-related behaviors in clinical samples: Current evidence and future directions. Journal of Clinical Psychology, 70(7), 631-643.

Hurtado, G., Hovey, J., \& Dueweke, A. (2019). Examining proximal risk factors for suicide in sample of Mexican adults in rehabilitation centers. Suicide and Life-Threatening Behavior, 49(1), 183-192.

Jobes, D. A., \& Joiner, T. E. (2019). Reflections on suicidal ideation. Crisis, 40(4), 227-230. 


\section{CHILDHOOD POLYVICTIMIZATION AND SUICIDE IDEATION}

https://doi.org/10.1027/0227-5910/a000615

Joiner, T. E. (2007). Why people die by suicide. Harvard University Press.

Joiner, T. E., Rudd, M. D., \& Rajab, M. H. (1997). The Modified Scale for Suicidal Ideation: Factors of suicidality and their relation to clinical and diagnostic variables. Journal of Abnormal Psychology, 106(2), 260-265.

Joiner, T. E., Van Ordern, K. A., Witte, T. K., \& Rudd, M. D. (2009). The interpersonal theory of suicide: Guidance for working with suicidal clients. American Psychological Association.

Karsberg, S., Charak, R., Lasgaard, M., Bramsen, R., Hansen, N., \& Elklit, A. (2019). Patterns of childhood abuse and dating violence victimization among early adolescents from Denmark: A latent class approach. Psychology of Violence, 9, 574-585.

Kennedy, T. M., \& Ceballo, R. (2013). Latino adolescents' community violence exposure: Afterschool activities and familismo as risk and protective factors. Social Development, 22(4), 663-682.

Lanier, P., Maguire-Jack, K., Walsh, T., Drake, B., \& Hubel, G. (2014). Race and ethnic differences in early childhood maltreatment in the United States. Journal of Developmental \& Behavioral Pediatrics, 35(7), 419-426. doi: $10.1097 / \mathrm{dbp} .0000000000000083$

Leong, F. T., \& Leach, M. M. (2008). Series in death, dying, and bereavement. Suicide among racial and ethnic minority groups: Theory, research, and practice. Taylor \& Francis.

Lev-Wiesel, R., \& Sternberg, R. (2012) Victimized at home revictimized by peers: Domestic child abuse a risk factor for social rejection. Child Adolescent Social Work

Journal, 29, 203-220. doi:10.1007/s10560-012-0258-0 


\section{CHILDHOOD POLYVICTIMIZATION AND SUICIDE IDEATION}

Li, S., Zhao, F., \& Yu, G. (2019). Childhood maltreatment and intimate partner violence victimization: A meta-analysis. Child Abuse and Neglect, 88, 212-224. doi:10.1016/j.chiabu.2018.11.012

Liu, C. H., Stevens, C., Wong, S. H. M., Yasui, M., \& Chen, J. A. (2018). The prevalence and predictors of mental health diagnoses and suicide among U.S. college students: Implications for addressing disparities in service use. Depression and Anxiety, 36, 8-17.

Ma, J., Batterham, P. J., Calear, A. L., \& Han, J. (2016). A systematic review of the predictions of the Interpersonal-Psychological Theory of Suicidal Behavior. Clinical Psychology Review, 46, 34-45. doi: 10.1016/j.cpr.2016.04.008

Miller, A. B., Esposito-Smythers, C., Weismoore, J. T., \& Renshaw, K. D. (2013). The relation between child maltreatment and adolescent suicidal behavior: a systematic review and critical examination of the literature. Clinical Child and Family Psychology Review, 16(2), 146-172. https://doi.org/10.1007/s10567-013-0131-5

Miller, A. B., Jenness, J. L., Oppenheimer, C. W., Gottleib, A. L. B., Young, J. F., \& Hankin, B. L. (2016). Childhood emotional maltreatment as a robust predictor of suicidal ideation: A 3-year multi-wave, prospective investigation. Journal of Abnormal Child Psychology, 45(1), 105-116. doi: 10.1007/s10802-016-0150-z

Molina, K. M., Little, T. V., \& Rosal, M. C. (2016). Everyday discrimination, family context, and psychological distress among latino adults in the United States. Journal of Community Psychology, 44(2), 145-165.

Nguyen, K. H., Kegler, S. R., Chiang, L., \& Kress, H. (2019). Effects of poly-victimization before age 18 on health outcomes in young Kenyan adults: Violence against children survey. Violence and Victims, 34, 229-242. 


\section{CHILDHOOD POLYVICTIMIZATION AND SUICIDE IDEATION}

Oakey-Frost, N., Tucker, R. P., \& Buckner, J. D. (2019). Ethnic identity and suicide risk among hispanic/latinx young adults: The impact of perceived burdensomeness and thwarted belongingness. Archives of Suicide Research. Advance online publication. doi.org/10.1080/13811118.2019.1670766

Preacher, K. J., \& Hayes, A. F. (2008). Asymptotic and resampling strategies for assessing and comparing indirect effects in multiple mediator models. Behavior Research Methods, 40(3), 879-891.

Puzia, M. E., Kraines, M. A., Liu, R. T., \& Kleiman, E. M. (2014). Early life stressors and suicidal ideation: Mediation by interpersonal risk factors. Personality and Individual Differences, 56, 68-72.

Rennison, C., \& Rand, M. R. (2003). Nonlethal intimate partner violence against women: A comparison of three age cohorts. Violence Against Women, 9(12), 1417-1428. h

Roeder, K. M., \& Cole, D. A. (2018). Simultaneous longitudinal examination of hopelessness, thwarted belongingness, and perceived burdensomeness as predictors of suicide ideation. Suicide and Life-Threatening Behavior, 49(4), 1058-1071.

Roley-Roberts, M. E., Zielinski, M. J., Hurtado, G., Hovey, J. D., \& Elhai, J. D. (2017).

Functions of nonsuicidal self-injury are differentially associated with suicide ideation and past attempts among childhood trauma survivors. Suicide and Life-Threatening Behavior, 47(4),450-460.

Sabina, C., Cuevas, C., \& Cotignola-Pickens, H. (2016). Longitudinal dating violence victimization among Latino teens: Rates, risk factors, and cultural influences. Journal of Adolescence, 47, 5-15.

Salinas, C., \& Lozano, A. (2019). Mapping and recontextualizing the evolution of the term 


\section{CHILDHOOD POLYVICTIMIZATION AND SUICIDE IDEATION}

Latinx. An environmental scanning in higher education. Journal of Latinos and Education, 18(4), 216-235.

Schönfelder, A., Hallensleben, N., Spangenberg, L., Forkmann, T., Rath, D., \& Glaesmer, H. (2019). The role of childhood abuse for suicidality in the context of the interpersonal theory of suicide: An investigation in German psychiatric inpatients with depression. Journal of Affective Disorders, 245, 788-797. https://doi.org/10.1016/j.jad.2018.11.063

Silva, C., Hurtado, G., Hartley, C., Rangel, J. N., Hovey, J. D., Pettit, J. W., Chorot, P., Valiente, R. M., Sandin, B., \& Joiner, T. E. (2018). Spanish translation and validation of the Interpersonal Needs Questionnaire. Psychological Assessment, 30(10), 21-37.

Silva, C., \& Van Orden, K. A. (2017). Suicide among Hispanics in the United States. Current Opinion in Psychology, 22, 44-49. https://doi.org/10.1016/j.copsyc.2017.07.013

Sterzing, P. R., Gartner, R. E., Goldbach, J. T., McGeough, B. L., Ratliff, G. A., \& Johnson, K. C. (2019). Polyvictimization prevalence rates for sexual and gender minority adolescents: Breaking down the silos of victimization research. Psychology of Violence, 9(4), 419430. https://doi.org/10.1037/vio0000123

Substance Abuse and Mental Health Services Administration (2019, August 20). Results from the 2018 National Survey on Drug Use and Health: Mental health detailed tables. U.S. Department of Health and Human Services, Office of Minority Health. https://www.samhsa.gov/data/report/2018-nsduh-detailed-tables

Tourangeau, R., \& Smith, T. W. (1996). Asking sensitive questions: The impact of data collection mode, question format, and question context. Public Opinion Quarterly, 60(2), 275-304. 


\section{CHILDHOOD POLYVICTIMIZATION AND SUICIDE IDEATION}

Turner, H. A., Finkelhor, D., \& Ormrod, R. (2010). Poly-victimization in a national sample of children and youth. American Journal of Preventive Medicine, 38, 323-330.

Van Orden, K. A., Cukrowicz, K. C., Witte, T. K., \& Joiner, T. E. (2012). Thwarted belongingness and perceived burdensomeness: Construct validity and psychometric properties of the Interpersonal Needs Questionnaire. Psychological Assessment, 24(1), $197-215$.

Van Orden, K. A., Witte, T. K., Cukrowicz, K. C., Braithwaite, S. R., Selby, E. A., \& Joiner, T. E. (2010). The interpersonal theory of suicide. Psychological Review, 117(2), 575-600.

Van Orden, K. A., Witte, T. K., Gordon, K. H., Bender, T. W., \& Joiner, T. E. (2008) Suicidal desire and the capability for suicide: Tests of the interpersonal-psychological theory of suicidal behavior among adults. Journal of Consulting and Clinical Psychology, 76(1), $72-83$. 


\section{CHILDHOOD POLYVICTIMIZATION AND SUICIDE IDEATION}

Table 1

Correlation between Childhood Polyvictimization, Perceived Burdensomeness, Thwarted

Belongingness, and Suicide Ideation among Hispanic Undergraduate Students

\begin{tabular}{lcccc}
\hline Variables & 1 & 2 & 3 & 4 \\
\hline 1. Polyvictimization & - & & & \\
2. Perceived burdensomeness & .320 & - & & \\
3. Thwarted belongingness & .190 & .480 & - & - \\
4. Suicide ideation & .270 & .576 & .393 & \\
\end{tabular}

Note. All coefficients are significant at $p<.001$. Polyvictimization scores are on a continuous scale ranging from 0 to 34 . 


\section{CHILDHOOD POLYVICTIMIZATION AND SUICIDE IDEATION}

Table 2

Significant Indirect Effects of Childhood Polyvictimization on Suicide Ideation Through

Perceived Burdensomeness and Thwarted Belongingness.

\begin{tabular}{rrcccc}
\hline Pathways & $B$ & $S E$ & $\beta$ & $95 \%$ CI $(B)$ & $95 \%$ CI $(\beta)$ \\
\hline PV $\square$ PB $\square$ SI & 1.895 & .488 & $.141^{* *}$ & 1.075 to 3.018 & .083 to .211 \\
PV $\square$ TB $\square$ SI & .344 & .134 & $.026^{*}$ & .132 to .701 & .010 to .051 \\
\hline
\end{tabular}

Note. $\mathrm{PV}=$ Polyvictimization. $\mathrm{PB}=$ Perceived burdensomeness. $\mathrm{TB}=$ Thwarted belongingness . $\mathrm{SI}=$ Suicide ideation

${ }^{*} p<.05,{ }^{* *} p<.001$ 
Figure 1.

Standardized co-efficient ( $\beta$ ) of Childhood Polyvictimization on Suicide Ideation Through Perceived Burdensomeness and Thwarted Belongingness

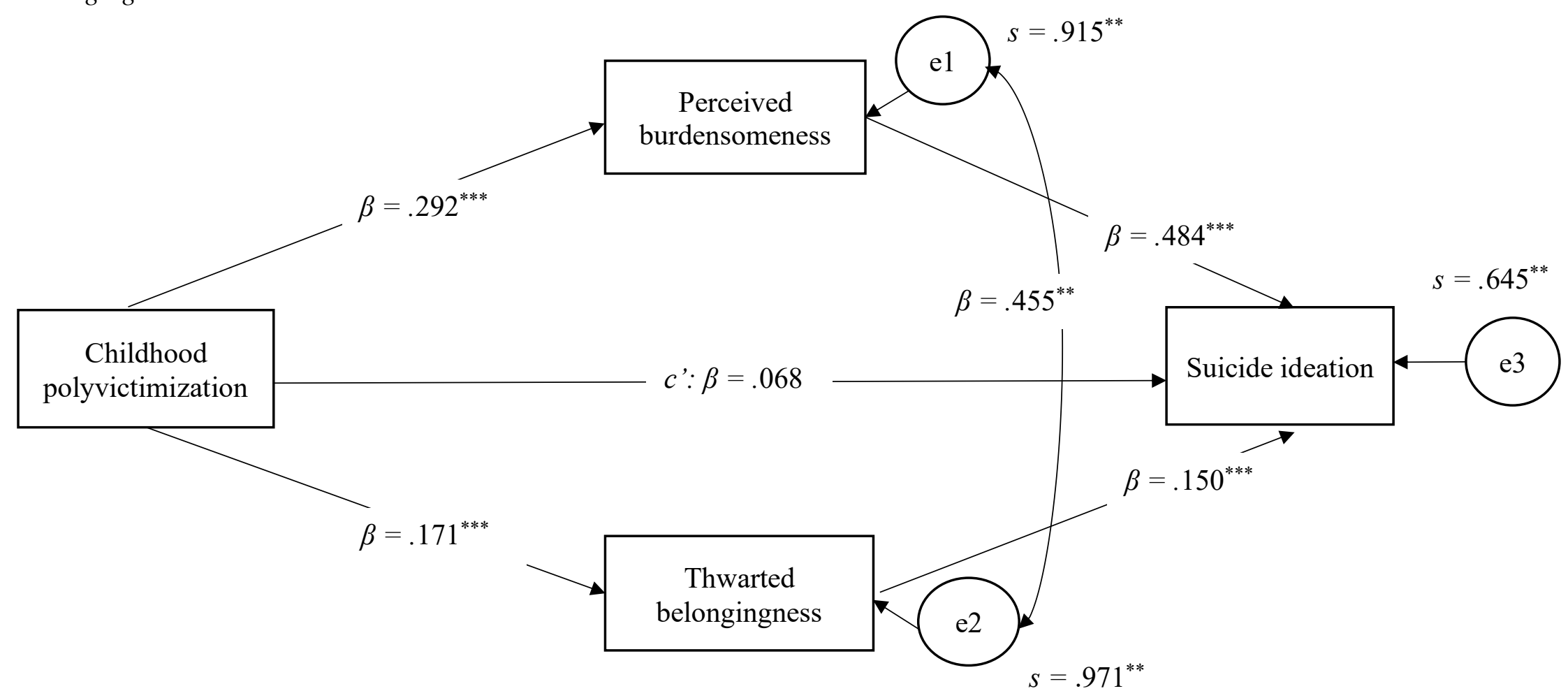

Note. ${ }^{*} p<.01 .{ }^{* *} p<.001$. Bootstrapping $=10,000$. 